\title{
Coulisses
}

Revue de théâtre

15 | Hiver 1997

Varia

\section{Rencontres Jeune Création, BVOJ 96}

1-18 juin 1996

\section{Bernard Billot}

\section{(2) OpenEdition}

Journals

Édition électronique

URL : http://journals.openedition.org/coulisses/5020

DOI : $10.4000 /$ coulisses.5020

ISSN : 2546-9460

Éditeur

Presses universitaires de Franche-Comté

\section{Édition imprimée}

Date de publication : 1 janvier 1997

Pagination : 23-24

ISSN : $1150-594 X$

\section{Référence électronique}

Bernard Billot, «Rencontres Jeune Création, BVOJ 96 », Coulisses [En ligne], 15| Hiver 1997, mis en ligne le 26 avril 2019, consulté le 23 octobre 2019. URL : http://journals.openedition.org/coulisses/ 5020 ; DOI : 10.4000/coulisses.5020

Ce document a été généré automatiquement le 23 octobre 2019.

Coulisses 


\title{
Rencontres Jeune Création, BVOJ 96
}

1-18 juin 1996

\author{
Bernard Billot
}

1 Organisé par la ville de Besançon, à l'initiative du service culturel, et depuis cette édition par l'adjoint à la culture Marcel Ferreol, BVOJ pour sa $11^{\mathrm{e}}$ édition a pris un nouveau départ, en s'appuyant plus encore sur des repères professionnels et un nouveau nom plus explicite : Rencontres Jeune Création.

2 Même si 1996 est restée une année de transition, la mise en place et la formalisation de critères hérités de dix ans d'expérience ont déjà porté leurs fruits :

- Soutien plus ciblé aux projets et hiérarchisation des aides ;

- Confirmation de la mise en place d'un environnement d'analyse (rôle fondamental de l'équipe du journal critique de Jacques Vingler) ;

- Refonte partielle du cadre d'accueil qui a permis de réduire les concurrences entre spectacles;

- Prolongement du travail pédagogique en amont avec les auteurs de projets : véritable suivi des propositions pour certains, de l'idée à la réalisation (mise en forme des dossiers, réunions et suivis techniques avec le personnel des salles de spectacles, aide à la formulation de véritables documents en direction de la presse...).

3 Cette somme de petites modifications et de confirmations d'orientations ont ainsi dès cette année permis de proposer au public 30 spectacles différents qui tous, dans leur démarche et leur réalisation, avaient objectivement leur place dans le cadre de Rencontres Régionales.

De l'expérience d'un stage réunissant le chanteur de "Ange» et trois groupes bisontins, en passant par l'énergie, la qualité et la justesse de "Gobe Mots " proposé par les élèves de l'école primaire La Bruyère, la création de musique contemporaine "Concert des Halles", jusqu'à la présence de la Cie Embarquez avec une création de théâtre de rue avant son départ pour Avignon, la programmation 96 a confirmé le bouillonnement culturel de Besançon et de sa région et l'existence de jeunes talents prometteurs. Il est à noter par exemple que le spectacle de la Cie L.T.S. adapté des Trois Sœurs de Tchékhov a reçu une récompense nationale en juin, alors que sa présence dans la manifestation était acquise dès le début de l'année civile à une période de forte 
adversité pour les jeunes comédiennes. Un exemple particulier qui tendrait à prouver à lui seul la justesse du regard de la manifestation sur la jeune création.

4 Tous les projets retenus sont arrivés à maturité et aux échéances fixées. Seul «Confidences » spectacle de danse contemporaine de la Cie Courant d'air a dû, pour des raisons techniques, être déplacé sur septembre, mais il s'est effectivement déroulé et a lui aussi trouvé son public.

5 Avec une nette dominante théâtrale, la manifestation 96 s'est déroulée sur dix lieux, majoritairement au Kursaal et sur 18 jours et non 10 comme les années précédentes pour réduire les concurrences. Toujours garant d'un équilibre entre ouverture, expérience et qualité, elle s'est donné un rôle essentiel de tremplin pour les Jeunes Compagnies.

6 L'objectif de ces rencontres est désormais bien établi: promouvoir les jeunes compagnies pour leur donner leur chance. Elles n'oublient pas cependant de poser quelques références avec la présence de professionnels reconnus, de s'intéresser à l'avenir avec la participations d'acteurs culturels en formation, (Conservatoire National de Région, Ecole Régionale des Beaux-Arts, Embarcadère), et de créer un véritable esprit de rencontres en associant des spectacles issus d'ateliers (pratique amateur, associatifs ou milieu scolaire), encadrés par les intervenants professionnels.

\section{Quelques repères sur l'édition 96}

\section{Des projets symboles}

« Le Bal perdu »- belle création malgré l'adversité.

«L.T.S.» - un projet primé nationalement réalisé essentiellement grâce aux Rencontres.

«Gobe-Mots» et «Eh t'as vu?»- justesse d'un travail ambitieux avec de jeunes acteurs.

«Cinéphalium» et «Je brasse...il Brassens» - maturité de projets musicaux théâtralisés.

\section{Un véritable état des lieux du Théâtre}

- présence de professionnels reconnus (Embarquez).

- Émergence de jeunes troupes (L.T.S. - comédiens du Bal Perdu).

- Qualité du travail d'ateliers (Embarcadère - Alcyon - Amanite Phalloïde - Théâtre Universitaire de Franche-Comté).

\section{Les rencontres}

- Vrai lieu de création et d'expérimentation.

- Vers des rencontres au sens plein du terme (journal critique - dialogue troupes et équipe critique - vers un bilan collectif).

\section{Une organisation}

- Lourde mais relativement rodée (travail préparatoire - Relation Technique - Suivi réel des projets jusqu'à échéance...). 


\section{B.V.O.J. en chiffres}

$11^{\mathrm{e}}$ édition

18 jours de spectacles

10 lieux à Besançon

30 spectacles différents (sans compter la pré-ouverture et le Festival des Petites Formes du CDN)

20 spectacles de théâtre

8 spectacles de musique

2 spectacles de danse

42 représentations

50 groupes, compagnies, associations, ateliers...

600 acteurs sur scène

1 à 153 acteurs par spectacle

40F maximum le prix de la place

et plus de 10000 spectateurs

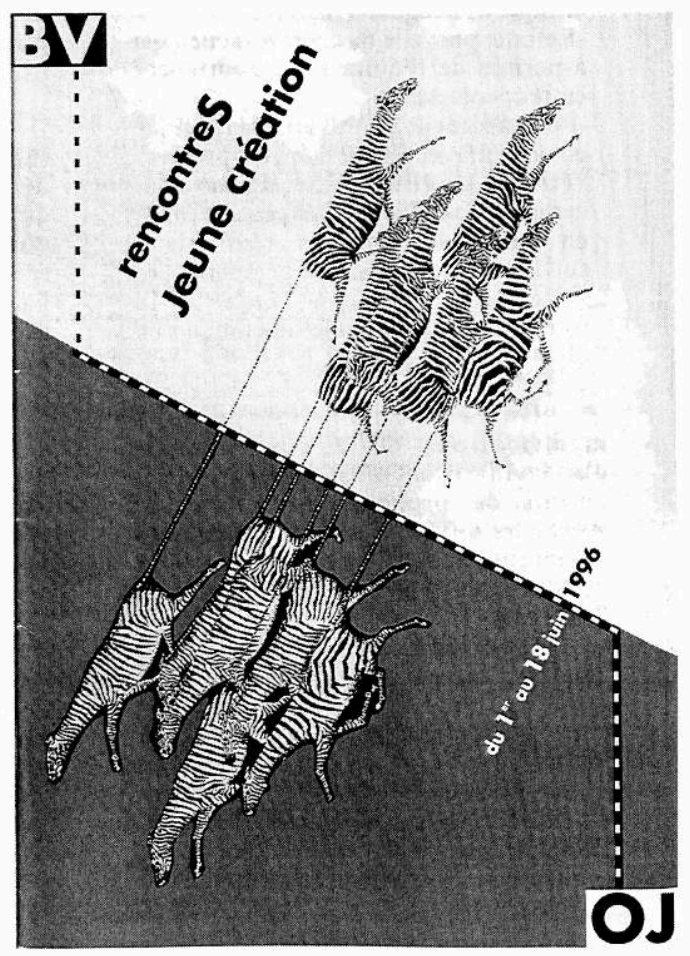

Composition Jean Loup Fontaine 